\section{Equivalências conceitual, semântica e instrumental: análises preliminares da versão em português (Brasil) da Male Body Dissatisfaction Scale (MBDS)}

\author{
Conceptual, semantic, and instrumental \\ equivalences: preliminary analysis of the \\ Brazilian Portuguese version of the \\ Male Body Dissatisfaction Scale (MBDS)
}

\section{Equivalencias conceptual, semántica e instrumental: análisis preliminares de la versión en portugués (Brasil) de la Male Body Dissatisfaction Scale (MBDS)}

Pedro Henrique Berbert de Carvalho 1 Maria Elisa Caputo Ferreira 1

Marcela Kotait ${ }^{2}$

Paula Costa Teixeira 2

Norman Hearst 3

Táki Athanássios Cordás 2

Maria Aparecida Conti 2

\footnotetext{
${ }^{1}$ Faculdade de Educação Física e Desportos,

Universidade Federal de Juiz de Fora, Juiz de Fora, Brasil.

2 Faculdade de Medicina,

Universidade de São Paulo,

São Paulo, Brasil.

3 School of Medicine,

University of California, San

Francisco, U.S.A.

Correspondência

P. H. B. Carvalho

Faculdade de Educação Física

e Desportos, Universidade

Federal de Juiz de Fora.

Rua José Lourenço Kelmer s/n,

Juiz de Fora, $M G$ 36036-900,

Brasil.

pedro.berbert@gmail.com
}

\begin{abstract}
This study aimed to describe the translation into Brazilian Portuguese and the analysis of conceptual, semantic, and instrumental equivalences and assessment of internal consistency of the Male Body Dissatisfaction Scale (MBDS). The study included the steps of (1) translation; (2) synthesis of translation; (3) back-translation; (4) review by experts - judges - analysis of the semantic and instrumental equivalences; (5) pre-test-assessment of verbal understanding of the instrument by specialists and male students; (6) analysis of the internal consistency by Cronbach's a coefficient. The MBDS was translated and adapted into Brazilian Portuguese maintaining its original 25 items, showed good verbal comprehension, and had an internal consistency coefficient of 0.92. The scale was translated and adapted with satisfactory results, thus confirming its initial psychometric qualities. The instrument requires further analysis of internal and external validity, and reproducibility.
\end{abstract}

Body Image; Eating Disorders; Men; Translating; Validation Studies

\section{Resumo}

O objetivo do estudo foi descrever o processo de tradução para o idioma português (Brasil), as análises das equivalências conceitual, semântica e instrumental e a avaliação da consistência interna da Male Body Dissatisfaction Scale (MBDS). Envolveu as seguintes etapas: (1) tradução; (2) síntese de tradução; (3) retrotradução; (4) revisão por peritos (juízes) - análise da equivalência semântica e instrumental; (5) pré-teste-avaliação da compreensão verbal do instrumento por especialistas e estudantes do sexo masculino; (6) análise da consistência interna por meio do coeficiente $\alpha$ de Cronbach. A MBDS foi traduzida e adaptada para o idioma português, mantendo seus 25 itens originais, e demonstrou ser de fácil compreensão, apresentando consistência interna de 0,92. A escala foi traduzida e adaptada obtendo resultados satisfatórios, o que comprova, assim, suas qualidades psicométricas iniciais. Necessita, ainda, de análises de validade interna e externa, e de reprodutibilidade.

Imagem Corporal; Transtornos da Alimentação; Homens; Tradução; Estudos de Validação 


\section{Introdução}

O público feminino apresenta maior insatisfação corporal e maior prevalência em relação aos transtornos alimentares quando comparado ao masculino ${ }^{1}$. No entanto, dados atuais demonstram que esse cenário está se alterando, com aumento na taxa de prevalência dos transtornos alimentares para os homens 2 .

A insatisfação ou distorção da imagem corporal pode estar presente em vários quadros psiquiátricos, mas é nos transtornos alimentares que seu papel sintomatológico é mais relevante ${ }^{3}$. Na psiquiatria, poucos transtornos têm uma distribuição por sexo tão assimétrica quanto os alimentares 4 , o que indica dificuldade no diagnóstico para homens ${ }^{4}$. Ademais, por não contemplarem questões específicas que representem as preocupações masculinas, os instrumentos utilizados para investigar a insatisfação, as crenças e os comportamentos de pacientes com essas patologias ficam com sua validade comprometida, enviesando os resultados 4 .

Cientes dessas limitações, Ochner et al. 5 desenvolveram a Male Body Dissatisfation Scale (MBDS), escala de 25 itens, variando de 1 (sempre/concordo fortemente) a 5 (nunca/discordo fortemente), com questões de sentido positivo (itens 1, 2, 3, 8, 11, 14, 15, 18, 19, 20, 21 e 23) e negativo (itens $4,5,6,7,9,10,12,13,16,17,22$, 24 e 25) 5 .

Nesse instrumento, outra avaliação sequencial é realizada para o mesmo item, nesse caso considerando o grau de importância (de 1 = sem importância a $10=$ grande importância) 5. Para esta avaliação, o valor de cada item é dividido por 10 e posteriormente multiplicado pela resposta dada a cada um (5), para, assim, obter o escore total da escala, que varia até 125 pontos 5 . Quanto maior o escore, maior a insatisfação corporal 5.

As evidências de validade iniciais da MBDS comprovam suas qualidades psicométricas, com satisfatória confiabilidade $(\alpha$-Cronbach $=0,93$ ), adequada validade para as medidas de autoestima (Rosenberg Self-Esteem Scale: $\mathrm{r}=-0,33 ; \mathrm{p}<$ 0,05) e autoestima corporal (Shape and Weight Based Self-Esteem Scale: $\mathrm{r}=0,53 ; \mathrm{p}<0,01 ;$ Body Esteem Scale: $\mathrm{r}=-0,29 ; \mathrm{p}<0,05)$ e para a autoavaliação da satisfação corporal - 100 pontos ( $\mathrm{r}=$ $-0,52 ; \mathrm{p}<0,01) 5$. Além disso, a escala apresenta satisfatória reprodutibilidade (teste-reteste) de $0,95(\mathrm{p}<0,0005) 5$.

O estudo original propõe a presença de três fatores, agrupados de acordo com os seguintes construtos: (1) musculature - musculatura; (2) definitions - definição; (3) relative standing/external evaluation - posicionamento relativo/avaliação externa 5 .
Uma vez que, no Brasil, contamos com um baixo número de instrumentos específicos para homens, o processo de adaptação transcultural permitirá a ampliação de pesquisas, o acompanhamento clínico de indivíduos com transtorno alimentar, bem como comparações entre diferentes populações por meio de pesquisas transculturais 6,7 .

O presente estudo tem como objetivo descrever o processo de tradução, as análises das equivalências conceitual, semântica e instrumental, bem como da consistência interna da MBDS, para a população masculina adulta brasileira.

\section{Métodos}

Este estudo utilizou procedimentos padronizados 8,9 e apoiou-se nas análises sugeridas por Herdman et al. 10 .

Solicitou-se a autorização do autor principal e, em seguida, procedeu-se à avaliação da equivalência conceitual 10, por meio de revisão de literatura, que indicou 11,12 a possibilidade da avaliação do construto na população brasileira.

$\mathrm{O}$ instrumento foi traduzido do inglês para o português por dois tradutores independentes (primeira etapa), os quais, em reunião, realizaram a síntese de tradução (segunda etapa). Esta foi, então, encaminhada a dois novos tradutores (terceira etapa) para a retrotradução. Participaram profissionais graduados, com especialização em língua inglesa: tradutor e intérprete ${ }^{8,9}$.

Para a análise da equivalência semântica (quarta etapa), foram reunidas todas as versões (tradução, síntese de tradução e retrotradução) e realizada reunião com os peritos. Em adição, foi avaliada a equivalência instrumental, em que foi verificada a necessidade, ou não, de alterações no formato, instrução e forma de aplicação da escala 10 , sendo elaborada uma versão para a realização do pré-teste (quinta etapa).

Esta quinta etapa constitui-se da análise da compreensão verbal do instrumento não só por especialistas $(n=10)$ no atendimento a pacientes com transtorno alimentar, como também por estudantes $(n=15)$. Para tanto, foi necessário adaptar a MBDS para o formato de escala verbal numérica 13 , apresentada em forma de escala Likert de pontos - de 0 (não entendi nada) a 5 (entendi perfeitamente e não tenho dúvidas), em que foi perguntado "Quanto você compreendeu do que foi perguntado em cada questão?”. Médias inferiores a três pontos foram consideradas como insuficientes para a compreensão verbal 13 .

Para análise da consistência interna (sexta etapa), a MBDS foi aplicada em uma amostra aleatória de 59 estudantes universitários (23,5 
anos $\pm 0,9$ anos), da cidade de São Paulo. Foram realizadas, ainda, análises descritiva (média e desvios-padrão) e inferencial ( $\alpha$-Cronbach).

Todos os indivíduos foram orientados sobre a finalidade da pesquisa e confirmaram sua participação voluntária por meio da assinatura do Termo de Consentimento Livre e Esclarecido. Este estudo está de acordo com as normas da Resolução no 196/96 do Conselho Nacional de Saúde e foi aprovado pelo Comitê de Ética em Pesquisa com Seres Humanos do Hospital das Clínicas, Faculdade de Medicina, Universidade de São Paulo (no protocolo: 0586/08).

\section{Resultados}

Tradução, equivalência semântica e instrumental

A tradução do instrumento foi considerada de fácil realização, no entanto modificações foram adotadas a fim de facilitar a compreensão da população-alvo. As mudanças propostas estavam relacionadas à presença de termos da língua nativa (coloquialismos, gírias ou jargões) e à possibilidade de mais de uma tradução para eles.

Os juízes decidiram pela mudança na forma de apresentação da escala e foi sugerida a aplicação em forma de quadro, em que cada item corresponderia a uma linha e cada opção de resposta a uma coluna. Fez-se necessária, portanto, a alteração das instruções aos respondentes, de "Circule uma das respostas abaixo de cada item..." para "Assinale uma das respostas, de acordo como seu sentimento atual em relação ao seu corpo" (Figura 1). Optou-se, porém, pela manutenção da sigla original da escala (MBDS) e pela adoção da tradução para o português Escala de Insatisfação Corporal Masculina.

\section{Compreensão verbal e consistência interna}

A avaliação dos especialistas e dos adultos indicou boa compreensão verbal (média superior a quatro pontos) e valores satisfatórios para a consistência interna ( $\alpha$ superior a 0,66 ) (Tabela 1 ).

\section{Discussão}

O presente estudo descreve uma das primeiras etapas de adaptação transcultural da MBDS. Teve o cuidado de realizar a tradução dando especial atenção ao sentido da linguagem original, buscou aproximação entre sentido conotativo e denotativo, possibilitou modificações e adaptações que respeitassem as particularidades da nossa cultura, a fim de que a escala possa ser compreendida por todos, indiscriminadamente $8,9,10$.

O grau de compreensão verbal, por parte tanto dos especialistas, quanto dos adultos, confirmou a equivalência semântica do instrumento ${ }^{10}$, com média superior a quatro pontos (máximo cinco pontos).

Optou-se pela manutenção da sigla original (MBDS), assim como já ocorreu em outros instrumentos validados para a população brasileira 14,15 , de modo que a escala seja facilmente identificada e reconhecida pela comunidade científica.

$\mathrm{Na}$ avaliação de sua estabilidade, o instrumento comprovou sua consistência interna ( $\alpha=$ $0,92)$, com valor similar ao encontrado no original $5(\alpha=0,93)$. Já para os três fatores que o compõem, os valores encontrados em nossa versão foram igualmente satisfatórios.

As alterações da imagem corporal, como a insatisfação com o corpo, estão presentes na população masculina adulta brasileira 11,12 e emergem como preocupações da área de saúde pública 11. Logo, a criação e a adaptação transcultural de escalas e questionários são importantes, pois disponibilizam materiais a serem aplicados considerando-se a realidade brasileira, garantindo, assim, que os dados aferidos expressem o que almejam mensurar.

O presente instrumento será importante para estudos científicos, epidemiológicos e clínicos, necessitando, ainda, de análises futuras que possam avaliar sua validade interna e externa, como também sua reprodutibilidade 8,9,10.

\section{Conclusão}

A MBDS foi traduzida e adaptada para o português apresentando adequadas qualidades psicométricas iniciais. 
Figura 1

Escala de Insatisfação Corporal Masculina (Male Body Dissatisfation Scale; MBDS).

Altura: $\_\mathrm{m} \longrightarrow \mathrm{cm}$.
Peso: $\_$kg.
Tipo corporal (escolha um): magro normal/estou na média sobrepeso musculoso

POR FAVOR, Leia cada questão com cuidado e responda honestamente. Todas as respostas são confidenciais.

Etapa A - Na linha que antecede cada item, classifique a importância do fato descrito.

Escolha um número de 1 a 10 ( 1 = sem importância a 10 = grande importância).

Etapa B - Depois disso, assinale uma das respostas, de acordo com o seu sentimento atual em relação ao seu corpo. Use as opções abaixo: 1- Concordo fortemente 2- Concordo parcialmente 3- Neutro 4- Discordo 5- Discordo fortemente

\begin{tabular}{|c|c|c|c|c|c|c|}
\hline \multicolumn{2}{|r|}{ Etapa A } & \multicolumn{5}{|c|}{ Etapa B } \\
\hline & $1=$ sem importância $10=$ grande importância & 1 & 2 & 3 & 4 & 5 \\
\hline 1. & $\begin{array}{l}\text { Estou feliz com a quantidade de músculos que tenho em relação à quantidade de gordura } \\
\text { do meu corpo }\end{array}$ & & & & & \\
\hline 2. & Outras pessoas acham que tenho um corpo bom & & & & & \\
\hline 3. & Eu tenho um peso adequado para minha altura & & & & & \\
\hline 4. & Eu gostaria de ter braços mais musculosos & & & & & \\
\hline 5. & Eu hesito em tirar a camiseta em público, pois as pessoas olharão para o meu corpo & & & & & \\
\hline 6. & Eu fantasio sobre ter mais músculos & & & & & \\
\hline 7. & Eu tenho pensamentos de insatisfação em relação ao meu corpo & & & & & \\
\hline 8. & No geral, acho que tenho um corpo atraente & & & & & \\
\hline 9. & Eu gostaria de ter o tronco mais em formato de " $\mathrm{V}$ " (triângulo invertido) & & & & & \\
\hline 10. & Eu gostaria de tornar-me mais tonificado para acentuar os músculos que tenho & & & & & \\
\hline 11. & Eu sou mais musculoso que a média dos homens da minha idade & & & & & \\
\hline 12. & Preocupo-me em ser mais musculoso & & & & & \\
\hline 13. & Eu gostaria de ter bíceps maiores & & & & & \\
\hline 14. & Acho que meus músculos peitorais são bem desenvolvidos & & & & & \\
\hline 15. & Eu tenho barriga de "tanquinho" & & & & & \\
\hline 16. & Se eu fosse mais musculoso, os outros me achariam mais atraente & & & & & \\
\hline 17. & Eu gostaria de perder mais gordura & & & & & \\
\hline 18. & Meu corpo aparenta ser saudável & & & & & \\
\hline 19. & Eu gosto de exibir meu corpo & & & & & \\
\hline 20. & A forma do meu corpo é um dos meus pontos positivos & & & & & \\
\hline 21. & Eu aparento conseguir levantar mais peso do que a média dos homens da minha idade & & & & & \\
\hline 22. & Eu gostaria de ter músculos mais definidos & & & & & \\
\hline 23. & Para as outras pessoas, meu corpo é sexualmente atraente & & & & & \\
\hline 24. & Penso que meu corpo é diferente do que acredito ser ideal & & & & & \\
\hline 25. & Eu gostaria de "malhar" e ter um corpo melhor & & & & & \\
\hline
\end{tabular}


Avaliação da compreensão verbal e consistência interna da Male Body Dissatisfaction Scale (MBDS). São Paulo, São Paulo, Brasil, 2011.

\begin{tabular}{|c|c|c|c|c|}
\hline \multirow[t]{5}{*}{ Questões } & \multirow{5}{*}{$\begin{array}{l}\text { Especialistas }(n=10) \\
\text { Grau de compreensão } \\
\text { Média (DP) }\end{array}$} & \multicolumn{3}{|c|}{ Adultos $(n=74)$} \\
\hline & & \multirow{4}{*}{$\begin{array}{c}\text { Grau de compreensão } \\
\text { Média (DP) } \\
(n=15)\end{array}$} & \multirow{3}{*}{\multicolumn{2}{|c|}{$\begin{array}{l}\text { Consistência interna } \\
\begin{array}{c}\alpha \text {-Cronbach } \\
(n=59)\end{array}\end{array}$}} \\
\hline & & & & \\
\hline & & & & \\
\hline & & & Importância & Concordância \\
\hline 4 & $5,0(0,0)$ & $4,3(1,4)$ & 0,88 * & 0,85 * \\
\hline 6 & $5,0(0,0)$ & $4,2(1,3)$ & & \\
\hline 7 & $5,0(0,0)$ & $4,3(1,2)$ & & \\
\hline 9 & $4,9(0,3)$ & $3,5(1,7)$ & & \\
\hline 12 & $4,9(0,3)$ & $4,0(1,4)$ & & \\
\hline 13 & $5,0(0,0)$ & $4,2(1,4)$ & & \\
\hline 16 & $4,9(0,3)$ & $3,7(1,5)$ & & \\
\hline 24 & $4,8(0,6)$ & $3,9(1,6)$ & & \\
\hline 1 & $4,9(0,3)$ & $4,0(1,1)$ & 0,87 ** & 0,66 ** \\
\hline 3 & $4,9(0,3)$ & $4,4(0,8)$ & & \\
\hline 10 & $4,6(0,7)$ & $3,6(1,7)$ & & \\
\hline 15 & $5,0(0,0)$ & $4,2(1,4)$ & & \\
\hline 17 & $5,0(0,0)$ & $4,3(1,0)$ & & \\
\hline 18 & $5,0(0,0)$ & $4,0(1,4)$ & & \\
\hline 20 & $4,4(0,9)$ & $3,6(1,8)$ & & \\
\hline 22 & $5,0(0,0)$ & $4,1(1,6)$ & & \\
\hline 25 & $4,8(0,4)$ & $3,9(1,5)$ & & \\
\hline 2 & $4,8(0,4)$ & $4,1(1,0)$ & $0,87 * \star \star$ & $0,80 * \star \star$ \\
\hline 5 & $4,9(0,3)$ & $4,4(0,8)$ & & \\
\hline 8 & $4,9(0,3)$ & $4,1(1,4)$ & & \\
\hline 11 & $5,0(0,0)$ & $3,8(1,7)$ & & \\
\hline 14 & $5,0(0,0)$ & $4,1(1,2)$ & & \\
\hline 19 & $4,9(0,3)$ & $4,0(1,6)$ & & \\
\hline 21 & $4,9(0,3)$ & $4,1(1,4)$ & & \\
\hline 23 & $5,0(0,0)$ & $3,9(1,7)$ & & \\
\hline Total & $4,9(0,3)$ & $4,0(1,4)$ & & \\
\hline
\end{tabular}

* Musculatura;

** Definição;

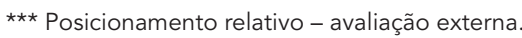




\section{Resumen}

El objetivo del estudio fue describir el proceso de traducción al idioma portugués (Brasil) de los análisis de las equivalencias conceptual, semántica e instrumental y la evaluación de la consistencia interna de la Male Body Dissatisfaction Scale (MBDS). El estudio contó con las siguientes etapas: (1) traducción; (2) síntesis de traducción; (3) retrotraducción; (4) revisión por especialistas (peritos) -análisis de la equivalencia semántica e instrumental; (5) pre-test-evaluación de la comprensión verbal del instrumento por especialistas y estudiantes del sexo masculino; (6) análisis de la consistencia interna por medio del coeficiente alfa de Cronbach. La MBDS fue traducida y adaptada al idioma portugués, manteniendo sus 25 ítems originales, $y$ demostró ser de fácil comprensión, presentando una consistencia interna de 0,92. La escala fue traducida y adaptada, obteniendo resultados satisfactorios, lo que comprueba, de este modo, sus cualidades psicométricas iniciales. Necesita, asimismo, análisis de validez interna y externa, y de reproductibilidad.

Imagen Corporal; Trastornos de la Conducta Alimentaria; Hombres; Traducción; Estudios de Validación

\section{Colaboradores}

P. H. B. Carvalho contribuiu na concepção do projeto, coleta e análise dos dados, redação e discussão do artigo. M. E. C. Ferreira colaborou na redação e revisão do artigo. M. Kotait participou na concepção do projeto, análise dos dados, redação e discussão do artigo. P. C. Teixeira e M. A. Conti contribuíram na concepção do projeto, análise dos dados, redação e discussão do artigo. N. Hearst e T. A. Cordás colaboraram na redação e revisão do artigo.

\section{Referências}

1. Treasure J, Claudino AM, Zucker N. Eating disorders. Lancet 2010; 375:583-93.

2. Striegel-Moore RH, Rosselli F, Perrin N, DeBar L, Wilson GT, May A, et al. Gender difference in the prevalence of eating disorder symptoms. Int J Eat Disord 2009; 42:471-4.

3. Saikali CJ, Soubhia CS, Scalfaro BM, Cordás TA. Imagem corporal nos transtornos alimentares. Rev Psiquiatr Clin (São Paulo) 2004; 31:164-6.

4. Melin P, Araújo AM. Transtornos alimentares em homens: um desafio diagnóstico. Rev Bras Psiquiatr 2002; 24:73-6.

5. Ochner CN, Gray JA, Brickner K. The development and initial validation of a new measure of male body dissatisfaction. Eat Behav 2009; 10:197-201.
6. Nunes MA, Barros FC, Olinto MTA, Camey S, Mari JD. Prevalence of abnormal eating behaviors and inappropriate methods of weight control in young women from Brazil: a population-based study. Eat Weight Disord 2003; 8:100-6.

7. Bowden A, Fox-Rushby JA. A systematic and critical review of the process of translation and adaptation of generic health-related quality of life measures in Africa, Asia, Eastern Europe, the Middle East, South America. Soc Sci Med 2003; 57:1289-306.

8. Guillemin F, Bombardier C, Beaton DE. Cross cultural adaptation of health-related quality of life measures: literature review and proposed guidelines. J Clin Epidemiol 1993; 46:1417-32. 
9. Beaton DE, Bombardier C, Guillemin F, Ferraz MB. Guidelines for the process of cross-cultural adaptation of self-report measures. Spine (Phila Pa 1976) 2000; 25:3186-91.

10. Herdman M, Fox-Rushby J, Badia X. A model of equivalence in the cultural adaptation of HRQoL instruments: the universalist approach. Qual Life Res 1998; 7:323-35.

11. Silva DAS, Nahas MV, Sousa TF, Del Duca GF, Peres KG. Prevalence and associated factors with body image dissatisfaction among adults in southern Brazil: a population-based study. Body Image 2011; 8:427-31.

12. Iriart JAB, Chaves JC, Orleans RG. Culto ao corpo e uso de anabolizantes entre praticantes de musculação. Cad Saúde Pública 2009; 25:773-82.

13. Abreu AM, Faria CDCM, Cardoso SMV, TeixeiraSalmela L. Versão brasileira do Fear Avoidance Beliefs Questionnaire. Cad Saúde Pública 2008; 24:615-23.
14. Conti MA, Slater B, Latorre MRDO. Validação e reprodutibilidade da Escala de Evaluación de Insatisfación Corporal para Adolescentes. Rev Saúde Pública 2009; 43:515-24.

15. Carvalho PHB, Conti MA, Cordás TA, Ferreira MEC. Tradução para o português (Brasil), equivalência semântica e consistência interna do Male Body Checking Questionnaire (MBCQ). Rev Psiquiatr Clin (São Paulo) 2012; 39:74-5.

Recebido em 29/Nov/2011

Versão final reapresentada em 28/Set/2012 Aprovado em 08/Out/2012 